\title{
CHANNEL ESTIMATION FOR LTE UPLINK SYSTEM BY PERCEPTRON NEURAL NETWORK
}

\author{
A. Omri ${ }^{1}$, R. Bouallegue ${ }^{2}$, R. Hamila ${ }^{3}$ and M. Hasna ${ }^{4}$. \\ 1 and 2 Laboratory 6’Tel @ Higher School of Telecommunication of Tunis. \\ ${ }^{1}$ omriaymen@qu.edu.qa, ${ }^{2}$ ridha.bouallegueesupcom.rnu.tn, \\ 3 and 4 Qatar University. \\ ${ }^{3}$ hamilalqu.edu.qa, ${ }^{4}$ hasnalqu.edu.qa
}

\begin{abstract}
In this paper, a channel estimator using neural network is presented for Long Term Evolution (LTE) uplink. This paper considers multiuser SC-FDMA uplink transmissions with doubly selective channels. This channel estimation method uses knowledge of pilot channel properties to estimate the unknown channel response at non-pilot sub-carriers. First, the neural network estimator learns to adapt to the channel variations then it estimates the channel frequency response. Simulation results show that the proposed method has better performance, in terms of complexity and quality, compared to the conventional methods least square (LS), MMSE and decision feedback and it is more robust at high speed mobility.
\end{abstract}

\section{KEYWORDS}

LTE, SC-OFDMA, Channel estimation, Perceptron.

\section{INTRODUCTION}

Third Generation Partnership Project Long Term Evolution (3GPP/LTE) is the name given to the project of the 3GPP to improve the UMTS mobile phone standard. LTE, which is also known as Evolved Universal Terrestrial Radio Access (E-UTRA), is a step toward the $4^{\text {th }}$ generation $(4 \mathrm{G})$ of mobile radio technologies to increase the capacity and the speed of mobile telephone networks.

The first version of LTE is documented in Release 8 of the 3GPP specifications. Release 8's air interface is assumed to use OFDMA (Orthogonal Frequency Division Multiple Access) for the downlink and SC-FDMA (Single Carrier Frequency Division Multiple Access) for the uplink [1].

The downlink transmission scheme for E-UTRA is based on conventional OFDM. In an OFDM system, the available spectrum is divided into multiple carriers, called sub-carriers, which are orthogonal to each other. Each of these sub-carriers is independently modulated by a low rate data stream. OFDM is used as well in WLAN, WiMAX and broadcast technologies like DVB. OFDM has several benefits including its robustness against multipath fading and its efficient receiver architecture. LTE Uplink uses SC-FDMA to keep a low peak to average power ratio (PAPR). SC-FDMA has similar throughput performance and complexity as OFDMA. Hence, similar to OFDMA, SC-FDMA is highly sensitive the Doppler shift, which destroy the subcarrier orthogonality and give rise to intercarrier interference (ICI).

Several channel estimation techniques have been proposed to overcome ICI in OFDM. To facilitate the estimation of the channel in an OFDM system (such as WiMax, WiFi, and 3.9/4G), known signals or pilots could be inserted in the transmitted OFDM symbol. 
In this paper we present a robust channel estimation using neural network for LTE uplink under high selectivity. The principle of this method is to use the information given by the reference signals to estimate the channel frequency response.

The remainder of the paper is organized as follows: In section II, the SC-FDMA transmission system and the multipath mobile radio propagation channel model are described in section III, three used channel estimation methods; Least Square (LS) [2], MMSE [3] and estimation with decision feedback [4] are presented, also the proposed neural network-based mobile radio channel estimation technique is introduced, its performance is analyzed via simulations and a comparative study, with the well-known estimation methods, in section IV. Finally, section V concludes this paper.

\section{SYSTEM MODEL}

Uplink LTE system is based on SC-FDMA air interface transmission scheme. Figuree.1 shows the baseband equivalent system model.

Let $\mathrm{b}$ denote the binary symbols of an uplink LTE system where 16-QAM, 64-QAM and QPSK modulations can be used to modulate b [1], an insertion of pilot symbols is necessary for the channel estimation. Before the IDFT block a DFT operation and mapping to subcarrier used to minimize the PAPR, the sub-carrier mapping determines which part of the spectrum that is used for transmission by inserting a suitable number of zeros at the upper and/or lower end

Let $\bar{X}=\left[X_{0} X_{1} \ldots X_{N_{c}-1}\right]^{T}$ and $\bar{Y}=\left[Y_{0, i} Y_{1, i} \ldots Y_{N_{c}-1}\right]^{T}$ denote the input data of IDFT block at the transmitter and the output data of DFT block at the receiver, respectively.

Let $\bar{h}=\left[h_{0} h_{1} \ldots h_{N_{c}-1}\right]^{T}$ and $\bar{n}=\left[n_{0} n_{1} \ldots n_{N_{c}-1}\right]^{T}$ denote the sampled channel impulse response and AWGN, respectively.

Also, the channel frequency response is given by

$$
\bar{H}=\operatorname{DFT}_{N}(\bar{h})=\underline{F} \bar{h},
$$

and the noise in frequency domain is represented by

$$
\bar{N}=\underline{F} \bar{n} .
$$

Define the input matrix $\underline{X}=\operatorname{diag}(\bar{X})$ and $\underline{F}$ is the DFT-matrix [5]

$$
\underline{F}=\left[\begin{array}{cccc}
W_{N}^{0,0} & W_{N}^{0,1} & \ldots & W_{N}^{0, N_{C}-1} \\
W_{N}^{1,0} & W_{N}^{1,1} & \ldots & \cdot \\
\cdot & \cdot & \cdot & \cdot \\
\cdot & \cdot & \cdot & \cdot \\
\cdot & \cdot & & \cdot \\
W_{N}^{N-1,0} & \ldots & & W_{N}^{N-1, N_{C}-1}
\end{array}\right]
$$

Where, $N$ is the FFT size and

$$
W_{N}^{i, k}=(1 / \sqrt{N}) \exp ^{-j 2 \pi(i k / N)} .
$$

Assuming that the cyclic prefix length is larger than the channel delay spread, the interference between the OFDM symbols can be eliminated. Therefore the OFDM received signal is expressed by

$$
\begin{aligned}
\bar{Y}=\operatorname{DFT}_{N}\left(\operatorname{IDFT}_{N}(\underline{X}) \otimes \bar{h}+\bar{n}\right) & =\underline{X} \underline{F} \bar{h}+\bar{N} \\
& =\underline{X} \bar{H}+\bar{N} .
\end{aligned}
$$


International Journal of Wireless \& Mobile Networks ( IJWMN ), Vol.2, No.3, August 2010

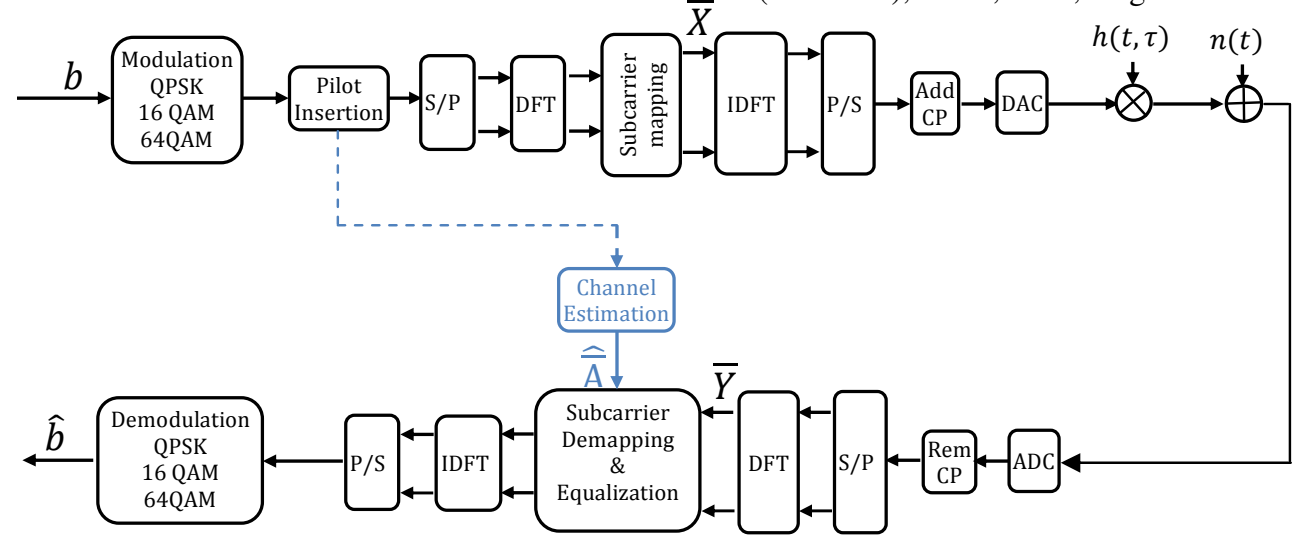

Figure 1 : Baseband equivalent system model.

$\stackrel{N_{\text {Symb }}^{\text {UL }}(=7 \text { OFDM symbols @ normal CP }) \quad 1 \text { slot }=15360 \times T_{s}=0.5 \mathrm{~ms}}{\longleftrightarrow}$

Cyclic Prefix

Figure 2 : OFDM symbol structure for normal cyclic prefix case [7]

The relationship between the input and the output for each OFDM subcarrier can be written as

$$
Y_{k, i}=H_{k, i} X_{k, i}+N_{k, i} \text {. }
$$

where, $H_{k, i}$ is the channel frequency response of the subcarrier $f_{k}$ given by

$$
f_{k}=f_{c}+k / T_{u} \text {. }
$$

$N_{k, i}$ are obtained by applying a DFT to the vector $\bar{n}$ where $n_{k, i}$ is the result of sampling $\mathrm{n}(\mathrm{t})$. a cyclic prefix is used for protect against multipath delay spread. Figure 2 shows the sevensymbols in a slot for the normal cyclic prefix case. Extended cyclic prefix is used in the case of high mobility, The time length is expressed in time units of $T_{s}=32.55 n s$, is the smallest sampling period in LTE standard, corresponding to the period of the sampling frequency $30.72 \mathrm{MHz}$.The length of the cyclic prefix is shown for the uplink in Table 1 [7].

Table 1: SC-FDMA cyclic prefix length [7].

\begin{tabular}{|l|l|}
\hline \multicolumn{1}{|c|}{ Configuration } & \multicolumn{1}{c|}{$\begin{array}{c}\text { Cyclic prefix } \\
\text { length } N_{C P, l}\end{array}$} \\
\hline Normal cyclic prefix & $\begin{array}{l}160 \text { for } l=0 \\
144 \text { for } l=1,2, \ldots, 6\end{array}$ \\
\hline Extended cyclic prefix & 512 for $l=0,1, \ldots, 5$ \\
\hline
\end{tabular}

The channel impulse response is given by [8]

$$
h(\tau, t)=\sum_{m=0}^{M-1} h_{m}(t) \delta\left(t-\tau_{m}\right),
$$

here, $M$ denotes the number of multipath, $h_{m}(t)$ and $\tau_{m}$ are the impulse response and the multipath delays of the channel, respectively.

The channel frequency response $H_{k, i}$ for the $k^{t h}$ subcarrier $f_{k}$ is given by the Fourier transform of the channel impulse response. 


\section{Channel estimation}

\subsection{LEAST SQUARE (LS)}

The principal of the channel least square estimator is minimizing the square distance between the received signal $\bar{Y}$ and the original signal $\underline{X}$ as follows [2]

$$
\begin{aligned}
\min _{H^{T}} J(H) & =\min _{H^{T}}\left\{|\bar{Y}-\underline{X} \cdot \bar{H}|^{2}\right\} \\
& =\min _{H^{T}}\left\{(\bar{Y}-\underline{X} \cdot \bar{H})^{T}(\bar{Y}-\underline{X} \cdot \bar{H})\right\},
\end{aligned}
$$

where, (. $)^{\mathrm{T}}$ is the conjugate transpose operator.

By differentiating expression (10) with respect to $\bar{H}^{\mathrm{T}}$ and finding the minima, we obtain

$$
\frac{\partial}{\partial \bar{H}^{T}} J(H)=-\underline{X}^{T} \bar{Y}+\underline{X}^{T} \underline{X} \bar{H}=0 .
$$

Finally, the LS channel estimation is given by [2]

$$
\widehat{\bar{H}}_{L S}=\underline{X}^{-1} \bar{Y}=\left[\begin{array}{llll}
\frac{Y_{0}}{X_{0}} & \frac{Y_{1}}{X_{1}} \ldots & \ldots & \frac{Y_{N_{c}-1}}{X_{N^{-1}}}
\end{array}\right]^{T} .
$$

In general, LS channel estimation technique for OFDM system has low complexity but it suffers from a high mean square error [2].

\subsection{MMSE ESTIMATOR}

The MMSE estimator employs the second-order statistics of the channel conditions to minimize the mean-square error.

Denote by , $\underline{R}_{h h}, \underline{R}_{H H}$ and $\underline{R}_{Y Y}$ the autocovariance matrix of $\bar{h}, \bar{H}$, and $\bar{Y}$, respectively, and by $\underline{R}_{h Y}$ the cross covariance matrix between $\bar{h}$ and $\bar{Y}$. Also denote by $\sigma_{N}^{2}$ the noise variance $\left\{|\bar{N}|^{2}\right\}$ Assume the channel vector $\bar{h}$ and the noise $\bar{N}$ are uncorrelated, this quantity are given by [3]:

$$
\begin{aligned}
\underline{R}_{H H}=E\left\{\bar{H} \bar{H}^{2}\right\} & =E\left\{(\underline{F} \bar{h})(\underline{F} \bar{h})^{H}\right\} \\
& =\underline{F} \underline{R}_{h h} \underline{F}^{H} . \\
\underline{R}_{h Y}=E\left\{\bar{h} \bar{Y}^{H}\right\} & =E\left\{\bar{g}(\underline{X} \underline{F} \bar{h}+\bar{N})^{H}\right\} \\
& =\underline{R}_{h h} \underline{F}^{H} \underline{X}^{H} . \\
\underline{R}_{Y Y} & =E\left\{\bar{Y} \bar{Y}^{H}\right\} \\
& =\underline{X} \underline{F} \underline{R}_{h h} \underline{F}^{H} \underline{X}^{H}+\sigma_{N}^{2} \underline{I}_{N} .
\end{aligned}
$$

Assume $\underline{R}_{h h}$ (thus $\underline{R}_{H H}$ ) and $\sigma_{N}^{2}$ are known at the receiver in advance, the MMSE estimator of $\bar{h}$ is given by [3].

$$
\hat{\bar{h}}_{M M S E}=\underline{R}_{h Y} \underline{R}_{Y Y}^{-1} \bar{Y}
$$

And $\widehat{\bar{H}}_{M M S E}$ is calculated as fellow [3]

$$
\begin{aligned}
\widehat{\bar{H}}_{M M S E} & =\underline{F} \widehat{\bar{g}}_{M M S E}=\underline{F}\left[\left(\underline{F}^{H} \underline{X}^{H}\right)^{-1} \underline{R}_{g g}^{-1} \sigma_{N}^{2}+\underline{X} \underline{F}\right]^{-1} \bar{Y} \\
& =\underline{F} \underline{R} g g\left[\left(\underline{F}^{H} \underline{X}^{H} \underline{X} \underline{F}\right)^{-1} \sigma_{N}^{2}+\underline{R}_{g g}\right] \underline{F}^{-1} \widehat{\bar{H}}_{L S} \\
& =\underline{R}_{H H}\left[\underline{R}_{H H}+\sigma_{N}^{2}\left(\underline{X} \underline{X}^{H}\right)^{-1}\right]^{-1} \widehat{\widehat{H}}_{L S}
\end{aligned}
$$


The MMSE estimator yields much better performance than LS estimators, especially under the low SNR scenarios. A major drawback of the MMSE estimator is its high computational complexity, especially if matrix inversions are needed each time the data in $\underline{X}$ changes.

\subsection{ESTIMATION With DECISION FEEDBaCK}

The OFDM Channel estimation with decision feedback uses the pilots to estimate the channel response $\widehat{\bar{H}}_{i}=\left\{\widehat{H}_{k, i}\right\}$ using LS or MMSE algorithms [4]. Here, $k=\{0, \ldots, N-1\}$ denotes the $k^{t h}$ subcarrier and $i$ the $i^{t h}$ symbol. For each coming symbol and for each subcarrier, the estimated transmitted symbol is found from the previous $\widehat{H}_{k, i}$ according to the formula

$$
\widehat{X}_{k, i+1}=\frac{Y_{k . i+1}}{\widehat{H}_{k, i}} .
$$

The estimated received symbols $\left\{\hat{X}_{k, i+1}\right\}$ are used to make the decision about the real transmitted symbol values $\left\{\tilde{X}_{k, i+1}\right\}$. The estimated channel response is updated by [4]

$$
\widehat{H}_{k, i+1}=Y_{k, i+1} / \tilde{X}_{k, i+1} \text {. }
$$

Consequently, $\widehat{H}_{k, i+1}$ is used as a reference in the next symbol, $i+2$, for the channel equalization.

\subsection{Proposed Neural Network Method}

\section{1) Principle}

The principle of the proposed estimation technique is inspired from the use of shape recognition in neural network. Figure 3 shows the principal of this method. The estimator uses the information provided by the pilots of sub channels to estimate the total channel frequency response. The estimation technique uses as input the information given by the pilots of each sub-channel. The input of the neural network, $\overline{\mathrm{P}}$, is defined by the following equation

$$
\begin{aligned}
\overline{\mathrm{P}}=\underline{X \mathrm{P}}^{-1} \overline{\mathrm{YP}} & =\left[\begin{array}{llll}
\frac{Y P_{0}}{X P_{0}} & \frac{Y P_{1}}{X P_{1}} & \ldots & \frac{Y P_{2 N_{p}-1}}{X P_{2 N_{p}-1}}
\end{array}\right]^{T} \\
& =\left[\begin{array}{lllll}
P_{0} & P_{1} & \ldots & P_{2 N_{p}-1}
\end{array}\right]^{T} .
\end{aligned}
$$

By making use of neural network, the following term will be estimated

$$
\begin{aligned}
\overline{\mathrm{A}}=\underline{\mathrm{X}}^{-1} \overline{\mathrm{Y}} & =\left[\begin{array}{llll}
\frac{Y_{0}}{X_{0}} & \frac{Y_{1}}{X_{1}} & \ldots & \frac{Y_{N_{c}-1}}{X_{N_{c}-1}}
\end{array}\right]^{T} \\
& =\left[\begin{array}{llll}
A_{0} & A_{1} & \ldots & A_{N_{c}-1}
\end{array}\right]^{T},
\end{aligned}
$$

$\underline{X}$ denote the transmitted OFDM symbols matrix, $\bar{Y}$ are the received OFDM symbols vector, $\underline{X}_{p}$ are the transmitted OFDM pilot, and $\overline{\mathrm{Y}}_{\mathrm{p}}$ are the corresponding received OFDM pilots.

The estimated output of the neural Network is given by

$$
\widehat{\bar{A}}=\bar{A}+\bar{e}=\left[A_{0}+e_{0} A_{1}+e_{1} \ldots A_{N_{c}-1}+e_{N_{c}-1}\right]^{T} .
$$

At the output of the channel equalization, we obtain the following expression

$$
\begin{aligned}
(\operatorname{diag}(\widehat{\bar{A}}))^{-1} \bar{Y} & =\left[\begin{array}{lll}
\frac{Y_{0}}{\widehat{A}_{0}} & \frac{Y_{1}}{\widehat{A}_{1}} \ldots \frac{Y_{N_{c}-1}}{\widehat{A}_{N_{c}-1}}
\end{array}\right]^{T} \\
& =\left[\frac{Y_{0}}{A_{0}+e_{0}} \frac{Y_{1}}{A_{1}+e_{1}} \cdots \frac{Y_{N_{c}-1}}{A_{N_{c}-1}+e_{N_{c}-1}}\right]^{T}
\end{aligned}
$$




$$
\begin{aligned}
& \simeq\left[\begin{array}{llll}
\frac{Y_{0}}{A_{0}} & \frac{Y_{1}}{A_{1}} & \ldots & \frac{Y_{N_{c}-1}}{A_{N_{c}-1}}
\end{array}\right]^{T},(\text { if } \bar{e} \approx \overline{0}) \\
& =(\operatorname{diag}(\overline{\mathrm{A}}))^{-1} \bar{Y} \\
& =(\operatorname{diag}(\overline{\mathrm{A}}))^{-1} \underline{X} \bar{A}, \operatorname{because}\left(\overline{\mathrm{A}}=\underline{X}^{-1} \overline{\mathrm{Y}}\right) \\
& =\underline{X}
\end{aligned}
$$

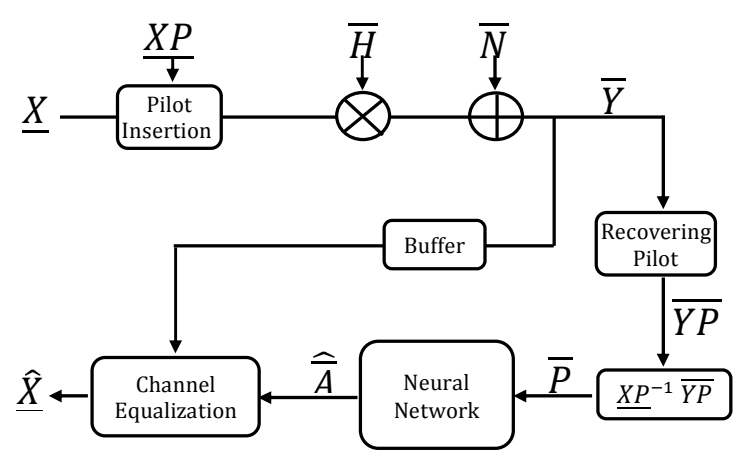

Figure 3 : Schematic diagram of the estimation phase.

The proposed method is based on Perceptron type of neural network having two separate phases i.e., learning phase and estimation phase.

The adopted architecture of neural network is carefully chosen after multiple tests of convergence by minimizing the learning time and keeping low implementation complexity, in order to increase the overall system performance.

The output of a single neuron is given by the following equation

$$
\hat{A}_{j}=f\left(\sum_{i=0}^{2 N_{p-1}} w_{j, i} P_{i}+b_{j}\right)
$$

Equation (21) can be presented in in matrix form as follows

$$
\hat{A}_{j}=f\left(\bar{W}_{j}^{T} \bar{P}+b_{j}\right) \text {. }
$$

Here, $\bar{W}_{j}^{T}=\left[\begin{array}{llll}w_{j, 0} & w_{j, 1} & \ldots & w_{j, 2 N_{p}-1}\end{array}\right], w_{j, i}$ is value of the synaptic weight connecting the stimulus $i$ to the neuron $j, P_{i}$ is the input stimulus, $\hat{A}_{j}$ is the neuron output in the range of $\left(0 \leq j \leq 2 N_{c}-1\right), f$ is the neuron output linear function and $b_{j}$ is the bias of the neuron $j$.

\section{2) Learning}

The estimator learning operation consists of changing the values of interconnection weights using learning algorithms for obtaining the desired performance. The learning algorithm in our proposed neural network is the efficient gradient backpropagation which minimizes the average square error between the outputs $\widehat{\bar{A}}$ and $\bar{A}$, by modifying the weights values. Figure 3 shows the principal of the learning phase.

The total squared error (for all output neurons $2 \mathrm{~N}_{\mathrm{c}}$ ) defining the network performance is given by:

$$
E=\frac{1}{N_{l}} \sum_{l=0}^{N_{l}-1} \sum_{j=0}^{2 N_{c}-1}\left(e_{j}^{l}\right)^{2}
$$


International Journal of Wireless \& Mobile Networks ( IJWMN ), Vol.2, No.3, August 2010

where, $e_{j}^{l}$ is the error on the $j^{\text {th }}$ neuron output and $l$ is the example of the training set, calculated by

$$
e_{j}^{l}=f\left(\bar{W}_{j}^{T} \cdot \bar{P}^{l}\right)-A_{j}^{l}=\hat{A}_{j}^{l}-A_{j}^{l}
$$

The weights $\bar{W}_{j}^{T}$ are updated with the following algorithm:

\section{Learning algorithm for the neural network}

1 - Initialize weights to low magnitude random values.

2 - Calculate the weight changes during one iteration:

$$
\overline{\Delta W}_{j}=-2 \mu \sum_{l=0}^{N_{l}-1} e_{j}^{l} f^{\prime}\left(\bar{W}_{j}^{T} \bar{P}^{l}\right) \bar{P}^{l} .
$$

3 - Update synaptic weights of each network:

$$
\bar{W}_{j}(k+1)=\bar{W}_{j}(k)+\overline{\Delta W}_{j}(k) .
$$

4 - If the error is large then it returns to Step 2, else we continue to Step 5.

5 - Desired performance of the neural network.

\section{3) Estimation}

After completing learning phase, the network uses the input data from the pilot channels $\bar{P}$ to estimate $\bar{A}$. Subsequently, the equalization followed by a decision estimate of the OFDMA symbols. For a single learning operation, the neural network estimate a large number of OFDM symbols in the range of 7000 symbols, corresponding to 50 radio frames LTE.

\section{SimULATION RESULTS}

We simulate an OFDM system with parameters shown in Table 3. These parameters are based on Uplink LTE system.

Table 3: Parameters of simulations [7], [9] and [10].

\begin{tabular}{|c|c|}
\hline Parameters & Specifications \\
\hline OFDM system & LTE/Uplink \\
\hline Constellation & QPSK \\
\hline Mobile Speed $(\mathrm{Km} / \mathrm{h})$ & $0: 50: 350$ \\
\hline$T_{S}(\mu \mathrm{s})$ & 72 \\
\hline$f_{c}(\mathrm{GHz})$ & 2.15 \\
\hline$\delta f(\mathrm{KHz})$ & 15 \\
\hline$B(\mathrm{MHz})$ & 5 \\
\hline Size of DFT/IDFT & 512 \\
\hline Number of users & 8 \\
\hline
\end{tabular}

In this part of our analysis, we are interested in comparing the proposed algorithm with the well-defined LS, MMSE and decision feedback. Figure 4 presents the variations in time and in frequency of the channel frequency response. The scenario of this simulation considers 8 users OFDMA uplink transmissions with doubly selective channels. Each user has a different mobile speed for 0 to $350 \mathrm{~km} / \mathrm{h}$. From these scenarios, we remark that the channel variations are large with the presence of high channel selectivity. Thus, robust algorithms for channel estimation are needed. 
The performance of the proposed estimator is compared with other estimation techniques, such as LS [2], MMSE [3] and decision feedback [4].

Figure 5 shows the variation of BER as a function of $E_{s} / \mathrm{N}_{0}$. Noticeably, the proposed method outperforms all other estimators, for example at $B E R=10^{-2}$ a gain of $15 \mathrm{~dB}$ over the decision feedback. This result prove the advantage and the capability of the neural network to adapt with the channel variation and give a better channel estimation to improve the service quality.

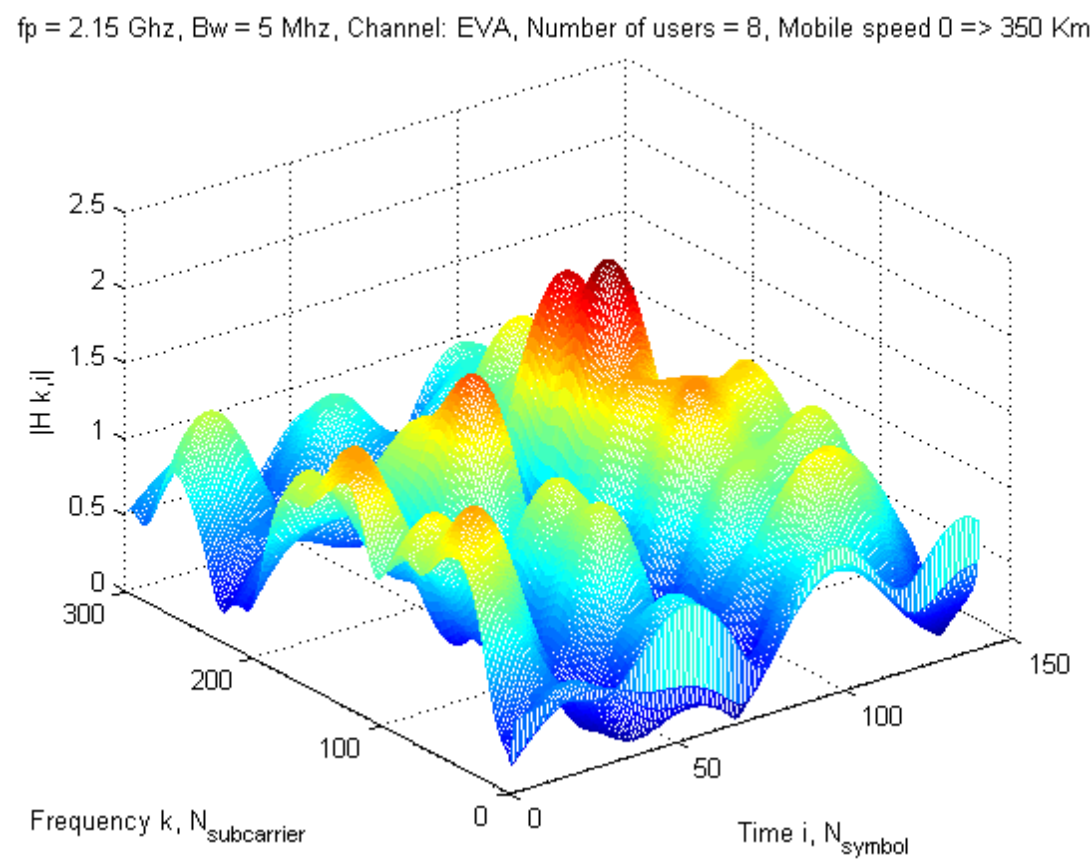

Figure 4 : variations in time and in frequency of the channel frequency response.

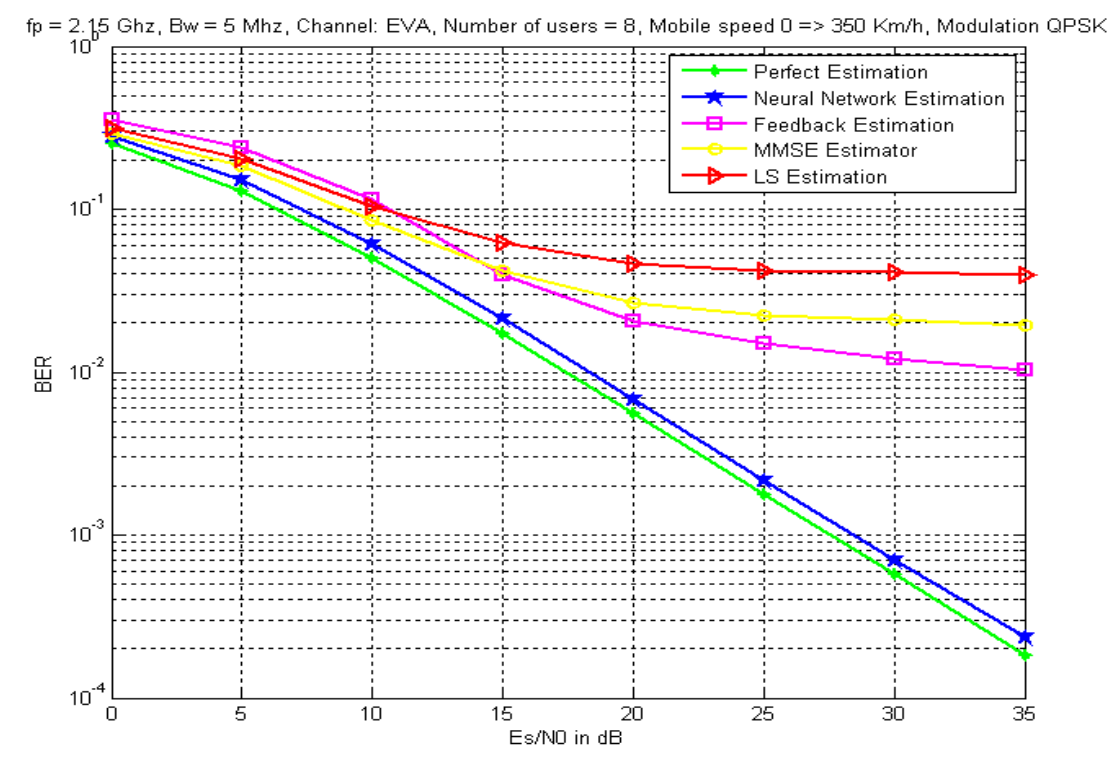

Figure 5 : BER as a function of Es/NO 


\begin{tabular}{|c|c|c|c|c|c|}
\hline \multirow{2}{*}{ Matrix } & \multirow[t]{2}{*}{ LS } & \multirow[t]{2}{*}{ MMSE } & \multirow{2}{*}{$\begin{array}{l}\text { Decision } \\
\text { Feedback } \\
\text { Estimation }\end{array}$} & \multicolumn{2}{|c|}{$\begin{array}{l}\text { Neural Network } \\
\text { Estimator }\end{array}$} \\
\hline & & & & Learning & Estimation \\
\hline Inversion $\mathrm{M}^{-1}$ & 1 & 2 & 2 & 2 & 0 \\
\hline Multiplication $\mathrm{M} \times \mathrm{N}$ & 1 & 3 & 2 & 1 & 1 \\
\hline Addition $\mathrm{M}+\mathrm{N}$ & 0 & 1 & 0 & 1 & 0 \\
\hline Soustratction $\mathrm{M}-\mathrm{N}$ & 0 & 0 & 0 & 1 & 0 \\
\hline \multirow{2}{*}{$\begin{array}{c}\text { Simulation duration/ } \\
\text { Symbol }\end{array}$} & 2.5 & 202.8 & 1.1 & $721.4 \mu s$ & $64.3 \mu s$ \\
\hline & $\mu s$ & $m s$ & $m s$ & \multicolumn{2}{|c|}{$785.7 \mu \mathrm{s}$} \\
\hline
\end{tabular}

Table 4. Complexity of Estimation Algorithms per OFDM Symbol

Table 4 shows the performance of our estimator in terms of simulation complexity in time and in terms of number of matrix operations. The proposed technique outperforms in terms of complexity compared to the considered estimators. In fact, in the estimation phase, the proposed method needs just one multiplication matrix and requires just $64.3 \mu$ s to estimate one OFDM symbol.

\section{Conclusions}

In this paper a new neural-network-based channel estimation technique for LTE uplink system is presented. The proposed channel estimation method uses reference signals of SC-FDMA system to estimate the variations of the channel frequency response in time and in frequency. This method is based on two phases, in the first phase, the proposed method learns to adapt to the channel variations, and in the second phase it estimates the channel frequency response.

First, the SC-FDMA transmission system and the multipath mobile radio propagation channel model are described. Then, three used channel estimation methods; Least Square (LS) [2], MMSE [3] and estimation with decision feedback [4] are presented, also the proposed neural network methods is described. After that, simulation scenarios considers multiuser SC-FDMA uplink transmissions with doubly selective channels is introduced. In this scenario we conceders 8 users with different mobile speeds from $O$ to $350 \mathrm{~km} / \mathrm{h}$ and the parameters of the 3GPP specification. Comparative study with well established techniques such as the LS, MMSE and decision feedback have been conducted. The results simulations, show clearly the high performance of the proposed methods when compared to these standard methods, such as, at $B E R=10^{-2}$ with the neural network methods we have a gain of $15 \mathrm{~dB}$ over the decision feedback. Also, the performance of the proposed estimator, in terms of computation complexity and number of required operations, are evaluated. Particularly, for a highly selective LTE Uplink system, the obtained results are very promising to improve the service quality in the LTE Uplink System.

\section{ACKNOWLEDGEMENTS}

This work was supported by Qatar Telecommunication under the project QUEX-Qtel09/10-10. 
International Journal of Wireless \& Mobile Networks ( IJWMN ), Vol.2, No.3, August 2010

\section{REFERENCES}

[1] M. Rumney, " LTE and the Evolution to 4G Wireless : Design and Measurement Challenges", Agilent Technologies Publication, 2009.

[2] C. Lim, D. Han," Robust LS channel estimation with phase rotation for single frequency network in OFDM", IEEE Transactions on Consumer Electronics, Vol. 52, pp. 1173 $1178,2006$.

[3] S. Galih, T. Adiono and A. Kurniawan, " Low Complexity MMSE Channel Estimation by Weight Matrix Elements Sampling for Downlink OFDMA Mobile WiMAX System”, International Journal of Computer Science and Network Securityb (IJCSNS), February 2010.

[4] A. Baynast, A. Sabharwal, B. Aazhang, "Analysis of Decision-Feedback Based Broadband OFDM Systems ”, Conference on Signals Systems \& Computers ACSSC, 2005.

[5] B. Karakaya, H. Arslan and H.Ali Cirpan, "Channel Estimation for LTE Uplink in High Doppler Spread ”, WCNC, 2008.

[6] J. Ketonen, M. Juntti and J. R. Cavallaro, " Performance-Complexity Comparison of Receivers for a LTE MIMO-OFDM System ", IEEE Transaction on Signal Processing, VOL. 58, NO. 6, JUNE 2010.

[7] 3rd Generation Partnership Project, “Technical Specification Group Radio Access Network; evolved Universal Terrestrial Radio Access (UTRA): Physical Channels and Modulation layer ", TS 36.211, V8.8.0, September 2009.

[8] Al-Naffouri. T.Y, Islam. K.M.Z, Al-Dhahir. N, Lu.S , “A Model Reduction Approach for OFDM Channel Estimation Under High Mobility Conditions ", IEEE Transaction on Signal Processing, Vol. 58, No. 4, April 2010.

[9] 3rd Generation Partnership Project, "Technical Specification Group Radio Access Network; Physical layer aspects for evolved Universal Terrestrial Radio Access (UTRA)", TR 25.814, V7.1.0, September 2006.

[10] 3rd Generation Partnership Project, "Technical Specification Group Radio Access Network; evolved Universal Terrestrial Radio Access (UTRA): Base Station (BS) radio transmission and reception”, TS 36.104, V8.7.0, September 2009. 


\section{Authors}

Aymen OMRI, was born in Tunisia, on November 29, 1983, he graduated in Telecommunications Engineering, from Aviation Academy of Borj El Amri, in Tunisia air force, June 2007. In June 2009 he obtained the masters degree of research in communication system of the School of Engineering of Tunis ENIT.

Currently he is a Ph.D student at the School of Engineering of Tunis. He is a researcher associate with Qatar Telecommunication QTel.

His research spans radio channel estimation and radio network planning in Wimax and LTE system,

Ridha BOUALLEGUE, is Professor at the National Engineering School of Tunis, Tunisia (ENIT), he practices at the Superior School of communication of Tunis (Sup'Com). He is founding in 2005, and Director of the Research Unit "Telecommunications Systems: 6'Tel@Sup'ComT. He is founding in 2005, and Director of the National Engineering School of Sousse. He received his PhD in 1998 then HDR in 2003. His research and fundamental development, focus on the physical layer of telecommunication systems in particular on digital communications systems, MIMO, OFDM, CDMA, UWB, WiMAX, LTE, has published 2 book chapters, 75 articles in refereed conference lectures and 15 journal articles (2009).

Ridha HAMILA, Senior Member of the Institute of Electrical and Electronic Engineers (IEEE), since 2003. Docent (Associate Professor), Institute of Communications Engineering, Department of Information Technology, Tampere University of Technology, Finland, and Associate Professor, College of Engineering, Qatar University, Qatar. He received the M.Sc. and Ph.D. Degrees in electrical engineering (EE) from Tampere University of Technology (TUT), Finland, in 1996 and 2002.

His research spans Channel Estimation for Accurate Personnel Positioning: WCDMA, GPS Signal Processing for All-Digital Receivers, Synchronization Techniques for Digital Receivers and Teager Energy Based Signal
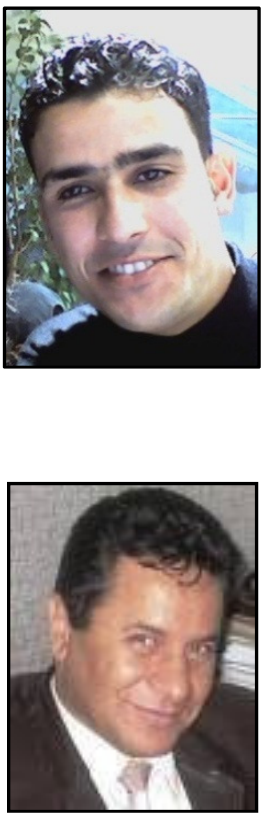
Processing

Mazen OMER HASNA, he received his BSc degree in 1994 from Qatar University, MS degree in 1998 from the University of Southern California and $\mathrm{PhD}$ degree in 2003 from the University of Minnesota, all in Electrical Engineering, majoring in communications engineering. In 2003, he joined the electrical engineering department at Qatar University as an assistant professor, and was appointed as the department head in 2005. In 2007, In July 2008, he was appointed as the dean of engineering. He has more than twenty publications in international journals and conferences, and is currently involved in several major research projects in the area of wireless

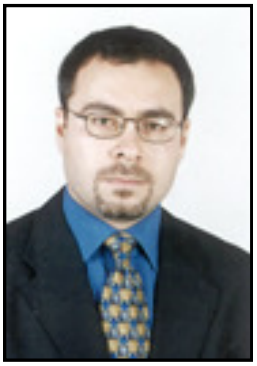
communications. 